\title{
George Dandin: Document social
}

La comédie George Dandin ou le mari confondu (1668) de Molière est une pièce de thêâtre particulièrement intéressante de plusieurs points de vue. D'une part, elle résume bien des courants généraux de la pensée de 1'époque, et pose, sous le voile du comique, bon nombre de problèmes dont les morales diverses du XVII ${ }^{\mathrm{e}}$ siècle se préoccupaient. D'autre part, son ambiguité même explique en partie les difficultés des critiques à saisir "le vrai Molière." Faut-il décider en faveur d'un Molière moraliste, observateur et critique de son siècle, inventeur de la comédie de vérité, 1 ou bien d'un Molière homme de thêâtre, technicien, héritier des traditions comiques, et nullement psychologue ou moraliste? ${ }^{2}$ Jusqu'où va l'illusion comique? Le ballet de Molière est-il, comme le veut Fernandez; "le saut dans 1'audelà, l'élan sans retour, sans réaccommodation factice à 1 'optique du monde" ? ${ }^{3} N^{\prime} y$ a-t-il aucun rapport entre la scène et la salle, aucun pont qui joigne le parterre et l'île enchantée du théâtre? Ou bien, au contraire, le thêatre est-il une manifestation de la réalité sociale?

Certes, la part de la convention comique est grande. Molière puisa le thème de George Dandin dans la Jalousie du barbouillé, créée sans doute vers 1646 , la plus vieille farce de son répertoire du temps des apprentissages provinciaux, et qui rejoint d'une ligne plus ou moins directe $1^{\prime}$ ancienne tradition des farces françaises et de la Commedia dell'arte italienne. L'intrigue de George Dandin, comme de 1a Jalousie, se réduit au motif central de l'action de presque toute farce, le retournement de la situation, le dupeur dupé, et s'enracine davantage dans 1 a convention tradi- 
tionnelle par son sujet principal archiconnu, la guerre sempiternelle entre les sexes. Les personnages sont également conventionnels: le triangle de mari rustre, de femme rusée, de jeune amant, secondés tous par leurs domestiques. Les jeunes triomphent toujours du vieux barbouillé, tyran jaloux qui brutalise sa jeune femme, mariée contre son gré. Le caractère conventionnel de la Jalousie est souligné par l'emploi des masques traditionnels et des visages barbouillés de farine de la Commedia dell'arte; vingt ans plus tard, le recours au ballet et à la chansọn accentuait le cọté chimérique de George Dandin, ${ }^{2}$ qui faisait partie du Grand Divertissement Royal de Versailles de juillet 1668 et qui se terminait joyeusement par une sorte de bacchanale pastorale. Mais les vingt ans qui séparent le Molière farceur de province du Molière comédien du roi ont opéré une certaine évolution, et George Dandin, malgré ses éléments de farce, se trouve, grâce à l'évolution sociale qui a eu lieu en même temps, enrichi de toute une expression dynamique et complexe de la réalité sociale de l'époque. C'est là que réside la signification majeure de cette pièce, car, comme le dit si bien R. Albanese, "le fait théâtral se montre tiraillé entre deux forces contradictoires: d'une part, 1 'exaltation des idéologies, des valeurs que des groupes épousent, et, d'autre part, la négation 6 implicite et radicale de ces mêmes idéologies." 6

Un des indices les plus révélateurs de la nouvelle signification acquise par cette ancienne farce refaite à la morale d'un nouveau public est le fait que cette pièce fort prisée par les invités au Grand Divertissement Royal, privée des intermèdes pour sa représentation devant le public à Paris sur le thêâtre du Palais-Royal la même année, n'obtint qu'un 7 succès médiocre et prit même un accent assez âpre. ${ }^{7}$ On se demande pourquoi depuis longtemps, et une des raisons principales est sans doute que, une fois supprimé tout 1'apparat pastoral et joyeux, la pièce apnarẩt dans tout le 
sérieux et l'actualité du conflit conjugal doublé du conflit irrémédiable entre les classes sociales et entre les codes moraux que ces classes opposées épousent. Le vieux barbon dont la jalousie excessive et ridicule était justement punie dans 1a Jalousie est devenu un bourgeois, pas trop mêchant au fond, qui est présenté comme ridicule précisément parce que c'est un bourgeois, dont 1 'infériorité naturelle se voit exagérée par la comparaison continuelle avec la "qualité" de sa femme et de ses beaux-parents. Ceux-ci, tout en le méprisant lui ont donné leur fille parce que Dandin était riche et que son argent "a servi à reboucher d'assez bons trous" (GD I.iv.). Selon certains, dans cette aventure, seul Dandin, aux yeux de Molière, 8 est coupable de folie et de manque d'honnêteté, cette dernière qualité étant la fidélité au rang qu'on tient dans le monde. 9 Un bourgeois qui n'est pas fidèle à sa bourgeoisie, c'est-à-dire à la modestie de sa condition et à une certaine infériorité sociale, 10 devient ridicule par sa prétention et invite les risées du public et le dédain de la noblesse aux rangs de laquelle il cherche à s'insinuer. Et, malgré $1^{\prime}$ axiome de certains que le héros comique ne se convertit jamais, 11 c'est ce que Dandin a vite appris; désormais complètement désabusé, il ne s'étonne plus de l'attitude de ses beaux-parents, et dès le début de la pièce il reconnâ̂t sa folie:

Que mon mariage est une leçon bien parlante à tous les paysans qui veulent s'élever au-dessus de leur condition, et s'allier, comme j'ai fait, à la maison d'un gentilhomme! . . . J'aurais bien mieux fait, tout riche que je suis, de m'allier en bonne et franche paysannerie que de prendre une femme qui se tient au-dessus de moi, s'offense de porter mon nom, et pense qu'avec tout mon bien je n'ai pas acheté la 
qualité de son mari.

\section{(GD I.i.)}

Mais, si Dandin a été assez malavisé pour accepter ce mariage comme une occasion de s'élever, il a fallu toutefois deux partis pour conclure le contrat, et les Sotenville, eux, sont également coupables en fait d'honnêteté. Au lieu de rester fidèles à leur rang haut et de continuer à dédaigner le paysan et le bourgeois enrichis, ils ont trahi leur code en cherchant comme gendre un homme qui leur était inférieur mais qui avait le grand avantage compensateur de la richesse. Ils ont imposé leur choix à leur fille malgré elle:

$$
\begin{aligned}
& \text { M'avez-vous, avant le mariage, demandé } \\
& \text { mon consentement, et si je voulais bien } \\
& \text { de vous? Vous n'avez consulté, pour cela, } \\
& \text { que mon père et ma mère; ce sont eux, } \\
& \text { proprement, qui vous ont épousé . }{ }^{\cdot} \\
& \text { (GD II.ii.). }
\end{aligned}
$$

et ainsi, le "bourgeois gentilhomme" et le gentilhomme malhonnête ont conclu ce marché qui crée une situation en réalité pathétique, confinant au scandale et même au tragique. En dépit de l'orgueil prétentieux et désormais pour ainsi dire illégitime des Sotenville, Molière ne songe pas à nous émouvoir contre ceux qui abusent de la différence des rangs, dit Bénichou, et la pièce entière est conçue de façon à égayer le public au dépens du mari bourgeois. D'ailleurs, presque tous les bourgeois sont ridicules chez Molière, et bien entendu, 1a représentation caricaturale du bourgeois et du paysan était une tradition littéraire, mais $1^{\prime}$ attaque continue de Molière contre les bourgeois avait des causes historiques et sociologiques bien réelles. 12

Revenons aux changements dans I'ordre social 
auxquels nous avons déjà fait allusion. Auerbach nous rappelle que dans la première moitié du XVII siècle, les forces culturelles qui régissaient la pensée du public ne venaient pas de la cour, mais de la ville, de la grande bourgeoisie et des salons.13 ( $L$ 'influence de la cour a commencé à prédominer seulement avec $I^{\prime}$ avènement du règne de Louis XIV, qui a dû mener une campagne continue pour assurer l'unité et la cohésion de la nouvelle culture.) Et dans le domaine de la morale sociale, la distinction bourgeoise entre $1 a$ raison et la déraison fonctionnait comme un mécanisme social, nous apprend Foucault, 14 et il y a eu vers 1650 1'établissement de nouvelles normes, essentiellement bourgeoises, dans I'intégration sociale. L'âge classique a commencé à appréhender la folie comme désorganisation de la famille. La structure familiale valait à la fois comme règle sociale et comme norme de la raison et devenait ainsi un des critères essentiels de la raison.

On assiste à cette époque à la grande confiscation de l'éthique sexuelle par la morale de la famille. Longtemps le mouvement "précieux" lui a opposé un refus dont 1 'importance morale fut considérable: 1'effort pour réveiller les rites de $I^{\prime}$ amour courtois et en maintenir l'intégrité par-delà les obligations du mariage, la tentative pour établir au niveau des sentiments un solidarité et comme une complicité toujours prêtes à $I^{\prime}$ emporter sur les liens de 1a famille, devaient finalement échouer devant le triomphe de la morale bourgeoise. L'amour est désacralisé par le contrat. 15

Ce n'était pas seulement dans 1 e domaine de 1 a sexualité que les exigences familiales bourgeoises 
devenaient rigoureuses; il s'agissait avant tout d'éviter que le patrimoine ne soit dilapidé ou qu'il ne passe dans des mains indignes. Ainsi les Sotenville se verraient justifiés dans leur choix de gendre car il s'agit de restaurer et de protéger la fortune de la famille. Et George Dandin aurait désormais le droit de tyranniser sa femme au nom de I'institution sacrée du mariage et de la famille.

Sous Louis XIV, cependant, ce sont de nouveau les attitudes et les habitudes de pensée de la noblesse qui s'imposent et qui deviennent les idées moyennes de I'époque, car le public bourgeois façonne ses goûts suivant ceux du beau monde. "Paris est le singe de la cour.16 Molière écrivait pour la cour et se montrait toujours partisan de la vue noble de la vie à 1 'encontre des habitudes bourgeoises. Mais dans $I^{\prime}$ aristocratie domestiquée par Louis XIV, la prétention à la grandeur commence à se perdre dans les appâts du plaisir et produit la notion de privilège, de supériorité gratuite et $q u$ 'on ne songe pas à justifier, qui prend la première place dans la vie noble au fur et à mesure que I'aristocratie cesse d'exercer une fonction sociale effective, devient en fait inutile, et ne peut désormais exiger qu'un respect de nature servile. Ainsi, 1'irrésistible préséance de 1 'amant de haut parage sur le mari légitime reproduit un genre de relations observable dans toute la société de ce temps-1à,17 et le thème du privilège noble ou du "droit de seigneur" qui est celui d'Amphitryon, et, retenu à l'échelle de la vie réelle, celui de 1 a Princesse d'E1ide et de Dom Juan, est aussi à $\overline{\text { la }}$ base de George Dandin, dont le personnage éponyme ne peut rien contre I'amant noble de sa femme:

De quoi s'avise-t-i1 aussi, d'être jaloux de sa femme, et de vouloir qu'elle soit à lui tout seul? C'est un impertinent, et monsieur le vicomte lui fait trop d'honneur. (GD III.i.) 
Le bourgeois est surtout ridicule en amour, où ses caractéristiques caricaturales traditionnelles-1'avarice, 1a jalousie, 1a tyrannie domestique-ne sont guère convenables au bel amour d'inspiration courtoise. Molière aime peindre le bourgeois qui veut posséder une femme comme il ferait une terre ou une cassette d'argent, et George Dandin paraît, aux yeux d'Angélique au moins, un jaloux forcené comme Arnolphe:

Comment, parce qu'un homme s'avise de nous épouser, il faut d'abord que toutes choses soient finies pour nous, et que nous rompions tout commerce avec les vivants! C'est une chose merveilleuse que cette tyrannie de messieurs les maris; et je les trouve bons de vouloir qu'on soit morte à tous les divertissements, et qu'on ne vive que pour eux.

$$
\text { (GD II.ii.) }
$$

Nous voilà amenés à un des problèmes principaux de la pièce George Dandin et de la société dont elle est née: la liberté des femmes. La tradition gauloise et farcesque est pleine de femmes débauchées, perfides, vicieuses, mais la misogynie était réellement et profondément enracinée dans la pensée de l'époque, et des enseignements presqu'aussi exagérés que ceux qu'un Arnolphe donnait à une Agnès triomphaient encore dans l'éducation ordinaire qu'on donnait dans les familles ou au couvent, et régissaient les moeurs communes.18 La préciosité, réincarnation de la courtoisie médiévale, essayait de combattre les institutions partiarcales et essentiellement bourgeoises qui violaient la liberté sociale et morale de la femme. Les précieuses s'attaquaient aux mariages arbitraires et aux rapports malhonnêtes qui en résultaient souvent. Ainsi, toute la 
situation de contrainte conjugale de George Dandin a pour cause le choix de mari que les parents d'Angélique lui ont imposé malgré sa jeunesse, et la brutalité et la tyrannie "bourgeoises" que ce mari se croit en droit d'exercer sur sa femme devenue possession, achetée par ses biens. Molière a protesté contre les moeurs et les préjugés ancrés dans la bourgeoisie et qui tenaient en esclavage tant de jeunes femmes, et, en cela, il s'est fait partisan des précieuses, ce qui, cependant, ne l'a pas empêché par la suite de s'emporter contre leurs excès ridicules. Le sujet quasi-universel de ses pièces est la lutte des femmes et des jeunes gens contre les entraves familiales, représentées le plus souvent par quelque vieillard, et le triomphe des jeunes sur la contrainte et les vieux préceptes familiaux et bourgeois démodés.

Molière, malgré l'effort de la critique du XIX $^{\mathrm{e}}$ siècle pour faire de lui le partisan du bon sens bourgeois, apparaît plutôt comme le porte-parole du bon ton noble. En s'attaquant à I'autorité paternelle et martiale, institution sacrée de la morale bourgeoise, il a pour lui l'opinion de la. cour et des salons. C'est une lutte qui met aux prises, encore une fois, deux courants littéraires, deux traditions dramatiques et romanesques où il entre une bonne part de convention livresque, deux traditions également anciennes et puissantes qui vivaient côte à côte depuis le moyen âge: la tradition courtoise et le courant d'esprit gaulois, le culte de la femme idéale et toute-puissante dont l'amour peut encourager les hommes à la vertu et à de belles actions héroĩques, et la veine burlesque de la femme perfide et querelleuse qui fait inévitablement des cocus. Et Molière, homme de thêâtre, savait tirer le maximum d'effet comique de cette opposition de traditions si bien ancrées dans la culture française. Mais il y a bien plus que cela dans son oeuvre. Elle se double d'une valeur idéologique au service d'une situation sociale bien 
rêelle. Le thêâtre de Molière est "un mode d'intégration culturelle" qui constitue un principe de cohésion sociale. 19 "Le ridicule déshonore plus que le déshonneur." 20 Dans George Dandin, le dramaturge essaie de ridiculiser les excès et les préjugés et bourgeois et aristocratiques; il montre du doigt d'un côté Dandin, bourgeois qui tâche de sortir de sa classe sociale, de l'autre côté les nobles malavisés qui, oubliant leur position et ayant besoin de rétablir leur fortune, troublent la hiérarchie en prenant un gendre dans la bourgeoisie. Angélique est la victime de cette transgression sociale, et celle-1à, quand elle se révolte à la fin, fait le désespoir de son mari. Sans doute bien des incidents pareils ont dû se passer dans la vie réelle de 1 'époque.

La richesse et I'influence bourgeoises étaient des forces que Louis XIV a dû combattre pour imposer son nouvel ordre. La bourgeoisie, qui suivait 1 'aristocratie et adoptait ses attitudes, mais qui tenait le vrai pouvoir politique et économique, ${ }^{21}$ devait être unie par des liens psychologiques et sociaux à la noblesse qui avait le prestige mais qui n'avait plus le pouvoir; et le moyen d'accomplir cette unité, c'était d'intégrer la bourgeoisie à la cour en la faisant aspirer à 1 'honnêteté, et par là de l'assujettir au pouvoir royal. Le théâtre de Molière apparaît donc comme une sorte de propagande négative, qui, en faisant éclater le ridicule des personnages qui ne savent pas s'intégrer dans $1 a$ belle société et qui troublent I'ordre social-- George Dandin et les Sotenville--prône I'établissement et le maintien de la paix et de 1 'unité auxquelles Louis XIV rêvait.

En conclusion, écoutons J. Duvignaud: "L'art de Molière doit davantage à des incitations situées à des niveaux différents dans 1 'échelle de 1 'expérience sociale qu'à une tradition continue agissant comme une impulsion unique." 22 On ne peut pas expliquer Molière par la farce ou la sotie du moyen 
âge. Il faut tenir compte de "la part prométhéenne de la réalité sociale," c'est-à-dire "ce secteur de la réalité sociale où les hommes pris collectivement ou individuellement entrevoient la possibilité de la transformation ou de l'éclatement des structures sociales par suite de I'action humaine concertée."23 On trouve chez Molière la conscience d'une action possible contre les structures sociales représentées dans George Dandin par le personnage éponyme et par les Sotenville. Le thêâtre de Molière, loin d'être la simple continuité des traditions ou le produit des conventions, a pour contexte la société réelle, une "expérience collective mouvante, créatrice, dominée par les rivalités des groupes, les conflits de la liberté et des contraintes étouffantes."24

ANNE AMARI PERRY EMORY UNIVERSITY

\section{NOTES}

${ }^{1}$ Daniel Mornet, Molière (Paris: Boivin, 1943), p. 189 .

2 René Bray, Molière homme de théâtre (Paris: Mercure de France, 1954), p. 340 .

${ }^{3}$ Ramon Fernandez, La Vie de Molière (Paris, 1938), cité par Robert J. Nelson, "L'Impromtu de Versailles Reconsidered," French Studies, 11 (1957), p. 313.

${ }^{4}$ Jean Duvignaud, Sociologie du théâtre: essai sur les ombres collectives (Paris: PUF, 1965), p.1. 
$5_{\text {Bray, p. } 338 .}$

${ }^{6}$ Ralph Albanese, Le Dynamisme de la peur chez Molière (University, Miss.: Romance Monographs, Inc., 1976), p. 10.

7Molière, Oeuvres complètes, éd. P.-A. Touchard (Paris: Seuil, 1962), p. 411.

${ }^{8}$ Paul Bénichou, Morales du grand siècle (Paris: Gallimard, 1948), pp. 175, 191 .

9 Erich Auerbach, Mimesis, trad. W.R. Trask (Princeton: Princeton U. Press, 1953), pp. 369-70; Albanese, p. 37.

${ }^{10}$ Bénichou, p. 174 .

${ }^{11}$ Nelson, p. 312 .

${ }^{12}$ Bénichou, pp. 175, 191.

${ }^{13}$ Erich Auerbach, "La cour et la ville," Scenes from the Drama of European Literature (New York: Meridian Books, 1959), pp. 138-39.

${ }^{14}$ Michel Foucault, Histoire de $1 \mathrm{a}$ folie à 1 'âge classique (Paris: Gallimard, 1972), pp. $92 \mathrm{ff}$.

${ }^{15}$ Ibid., p. 104 .

${ }^{16}$ La Bruyère, Les caractères, De la ville, $\$ 15$. 
17 Bénichou, p. 166.

${ }^{18}$ Ibid., p. 189.

${ }^{19}$ Albanese, p. 203.

${ }^{20}$ La Rochefoucauld, Maximes, $\$ 326$.

${ }^{21}$ Auerbach, Scenes, pp. 162-74.

22 Duvignaud, p. 32 .

${ }^{23}$ Georges Gurvitch, Dialectique et sociologie (Paris: Flammarion, 1962), p. 224, cité par Duvignaud, p. 34 .

${ }^{24}$ Duvignaud, p. 36. 